\title{
FOSS4G DATE ASSESSMENT ON THE ISPRS OPTICAL STEREO SATELLITE DATA: A BENCHMARK FOR DSM GENERATION
}

\author{
M. Di Rita ${ }^{\mathrm{a}, *}$, A. Nascetti ${ }^{\mathrm{a}}$, M. Crespi ${ }^{\mathrm{a}}$ \\ ${ }^{a}$ Geodesy and Geomatics Division, DICEA, University of Rome "La Sapienza”, Italy - \\ (martina.dirita, andrea.nascetti, mattia.crespi)@uniroma1.it
}

KEY WORDS: Cartosat-1, Worldview-1, DSM, Matching, Accuracy, FOSS4G

\begin{abstract}
:
The ISPRS Working Group 4 Commission I on "Geometric and Radiometric Modelling of Optical Spaceborne Sensors", provides a benchmark dataset with several stereo data sets from space borne stereo sensors. In this work, the Worldview-1 and Cartosat-1 datasets are used, in order to test the Free and Open Source Software for Geospatial (FOSS4G) Digital Automatic Terrain Extractor (DATE), developed at Geodesy and Geomatics Division, University of Rome "La Sapienza", able to generate Digital Surface Models starting from optical and SAR satellite images. The accuracy in terms of NMAD ranges from 1 to $3 \mathrm{~m}$ for Wordview-1, and from 4 to $6 \mathrm{~m}$ for Cartosat-1. The results obtained show a general better 3D reconstruction for Worldview-1 DSMs with respect to Cartosat-1, and a different completeness level for the three analysed tiles, characterized by different slopes and land cover.
\end{abstract}

\section{INTRODUCTION}

One of the most important application of remote sensing is the generation of Digital Surface Models (DSMs), that have a large relevance in many engineering, environmental, Earth sciences, safety and security applications. The fully automatic generation of DSMs is still an open research issue. The International Society for Photogrammetry and Remote Sensing (ISPRS), in order to assess the accuracy and reliability of current methods for DSMs generation from images acquired by different platforms, provides benchmark datasets with several stereo images from high and very high resolution satellite sensors, together with ground truth data. The Worldview-1 and Cartosat-1 datasets from the ISPRS Matching Benchmark (Reinartz et al. 2010) are used in this research, in order to test the Free and Open Source Software for Geospatial (FOSS4G) Digital Automatic Terrain Extractor (DATE), developed at Geodesy and Geomatics Division, University of Rome "La Sapienza".

As a matter of fact, in recent years, new satellite platforms, with different characteristics, able to acquire stereo images have been launched and this benchmark could be interesting also considering the different acquisition procedure performed by the two sensors. In particular, Cartosat- 1 features two CCD lines for alongtrack acquisitions and exhibits a Ground Sample Distance (GSD) of $2.5 \mathrm{~m}$. No special manoeuvres of the satellites are necessary and long stereo stripes can be acquired in a short time span. On the contrary, the new generation of very high resolution sensors, like Worldview-1, features very agile manoeuvring, that allows stereo data acquisition within the same orbit, by pointing at the same area from different positions, using the CCD line combination.

\section{DSM GENERATION: DATE STRATEGY}

In this work, the results obtained with DATE on the above mentioned datasets are presented and assessed. DATE is a FOSS4G, conceived as an OSSIM (Open Source Software Image Map) plugin, whose development started in summer 2014 in the framework of 2014 Google Summer of Code, having as early purpose a fully

\footnotetext{
${ }^{*}$ Corresponding author
}

automatic DSMs generation from stereo high resolution optical satellite imagery acquired by the most common sensors. Nowadays, it is also able to handle multi-view acquisitions and SAR imagery. The implemented tool is based on a hybrid procedure, whereby photogrammetric and computer vision algorithms are mixed in order to take the best of both. As regards the dense matching algorithm, for example, the Semi Global Matching as implemented in the OpenCV library (Dröppelmann et al. 2010) is exploited. The main idea is based on the development of a fully automatic, efficient, precise and accurate tool for DSM generation from satellite imagery, paying special attention in finding a solution for the epipolar resampling for images acquired by optical and SAR satellite sensors, for which it is not straightforward the epipolar geometry achievement, due to their multiple projection centers.

The epipolar geometry achievement is carried out in the object space thanks to an images ground projection, and it is based on a roto-translation transformation model for Ground quasi-Epipolar Images (GrEI) generation and on a coarse-to-fine pyramidal scheme adopted. The GrEI are the core of this original strategy for DSMs generation from satellite imagery, able to return a reliable and accurate solution. As a matter of fact, the strategy for the epipolar resampling for satellite imagery, relies on a preliminary ground projection using an a-priori DSM (such as SRTM, a coarse and freely available global DSM), thanks to which the search space for the successive dense matching is significantly restricted. Then, an approximate rotation is computed through Rational Polynomial Coefficients RPCs, in order to align the images in the disparity prevailing direction. Afterwards, a rototranslation is estimated, thanks to a set of well-identified Tie Points, and applied, to refine the images relative orientation and to achieve the epipolar geometry: the obtained images can act as GrEI and can undergo a dense image matching procedure. Furthermore, in order to be able to keep the transversal parallax errors well below the imagery resolution, a coarse-to-fine pyramidal scheme is adopted: this allows to iteratively refine the a-priori DSM until the desired final resolution is achieved. The final DSM at each pyramidal level is the input for the next pyramidal level, acting as the a-priori DSM. In this way, at lower resolution it is possible to detect larger structures whereas at higher resolution small details are progressively added to the already obtained DSM. 
Since the disparities are computed in the object space, it is sufficient to sum them to the coarse DSM exploited as a reference for the initial projection, to obtain the final DSM. But first, the obtained local pixel disparity values have to be converted into height corrections with respect to the used a-priori DSM through an efficient procedure based on the computation of a disparity-to-height conversion factor $\left(C_{\text {factor }}\right)$, exploiting RPCs-based orientation model (Figure 1). A deeper detailed analysis of DATE methodology and functioning can be found in (Di Rita et al. 2017)

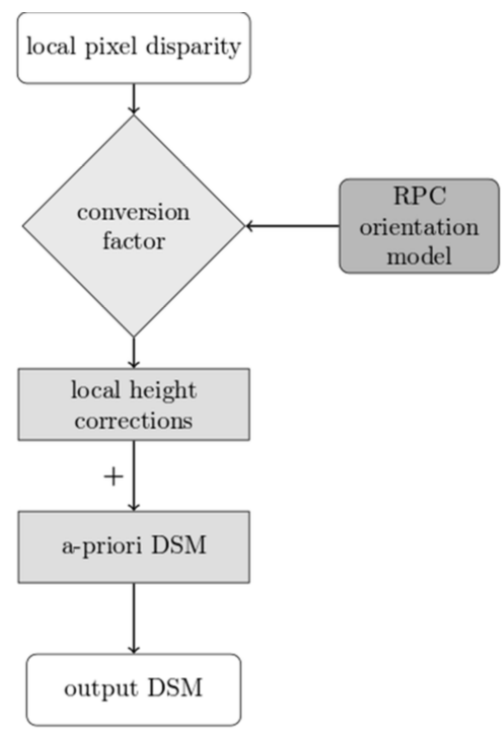

Figure 1: General workflow from pixel disparity to generated DSM

Relying on this strategy for epipolarity resampling, an efficient, automatic and open source software (freely available at the URL of the GitHub repository https://github.com/martidi/opencv_dsm/tree/imageStack) for DSM generation from optical and SAR satellite imagery, has been developed and implemented in C++ programming language. Thanks to the approach used for DSM generation starting from the raw images, DATE presents a great advantage in terms of efficiency, being in general about 3 times faster than other well-known commercial software packages (Di Rita et al. 2017; Fratarcangeli et al. 2016). A negligible processing time has been witnessed for the DSMs generation starting from the dataset of this benchmark (about one minute).

\section{RESULTS EVALUATION}

\subsection{DATASET}

The analysed regions are in Catalonia, Spain, and include three test areas, covering city areas, rural areas and forests, in flat and medium undulated terrain as well as steep mountainous terrain. The resulting DSMs are evaluated against the first pulse returns of the LiDAR reference dataset, provided by the Institut Cartografic de Catalunya (ICC).

Three small tiles (each of a $4 \mathrm{~km} \times 4 \mathrm{~km}$ size) are the object of this benchmark (Figure 2 and Table 3), in order to investigate different land cover and slopes.

The reference data consist of color orthoimages with a spatial resolution of $0.50 \mathrm{~m}$ as well as an airborne laser scanning point cloud (first pulse and last pulse) with approximately 0.3 points

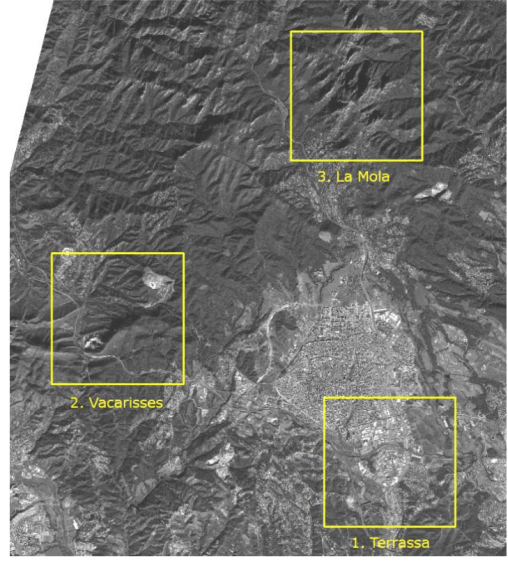

Figure 2: Overview of the three test areas (Reinartz et al. 2010)

\begin{tabular}{|c|c|c|}
\hline Test area & $\begin{array}{l}\text { Lower left } \\
\text { (UTM31N, } \\
\text { WGS84) }\end{array}$ & Area type \\
\hline 1. Terrassa & $\begin{array}{l}417400 \mathrm{E} \\
4597300 \mathrm{~N}\end{array}$ & City, industrial, residential \\
\hline 2. Vacarisses & $\begin{array}{l}409100 \mathrm{E} \\
4601700 \mathrm{~N}\end{array}$ & $\begin{array}{l}\text { Wooded hills, quarry, waste } \\
\text { dump }\end{array}$ \\
\hline 3. La Mola & $\begin{array}{l}416400 \mathrm{E} \\
4608600 \mathrm{~N}\end{array}$ & $\begin{array}{l}\text { Steep mountainous terrain, } \\
\text { forests }\end{array}$ \\
\hline
\end{tabular}

Figure 3: Test areas characteristics and position (in UTM 31N)

per square meter. ICC has also provided 143 oriented DMC images with a ground resolution of $25 \mathrm{~cm}$ and $60 \%$ forward overlap and $50 \%$ side overlap, covering all three test sites.

Cartosat-1 stereo pair, acquired on the 5th of March 2008, shows an intersection angle of $32^{\circ}$. A Worldview- 1 stereo pair, acquired on the 29th August 2008, with a stereo angle of the pair is $35^{\circ}$, is used.

\subsection{ASSESSMENT PROCEDURE}

As required by the benchmark guidelines, as far as concerns the Worldview-1 images, with a $0.5 \mathrm{~m}$ native GSD, the DSMs are produced at $1.0 \mathrm{~m}$ resolution. On the contrary, as regards the Cartosat-1 data, with a $2.5 \mathrm{~m}$ native GSD, the DSMs are generated at $5.0 \mathrm{~m}$ GSD.

The DSMs generated have been assessed without further processing, in order to evaluate the software behaviour as a whole: no 3D shift with respect to the reference DSM nor filtering refinement has been applied.

In order to assess the results, in addition to the standard statistical parameters usually used for the global DSMs assessment ((Rodriguez et al. 2006; Gesch et al. 2014), also more robust statistics have been computed. These include the median as a robust estimate for the mean height difference, the Normalized Median Deviation (NMAD) as a robust estimate of the standard deviation, and $68 \%$ and $95 \%$ percentiles of the absolute error.

In this work, the following significant statistical parameters are considered:

- $\overline{\Delta Z}$ : mean value of the height discrepancies $\Delta Z$ 


\begin{tabular}{l|cccccc} 
Test Area & Mean & Median & Std.Dev & NMAD & LE68 & LE95 \\
\hline Terrassa & 3,13 & 3,22 & 5,05 & 4,43 & 4,45 & 10,03 \\
Vacarisses & 0,33 & 0,51 & 6,01 & 5,36 & 5,43 & 11,99 \\
La Mola & $-7,88$ & $-7,55$ & 7,81 & 6,02 & 6,28 & 14,66
\end{tabular}

Table 1: Cartosat-1 assessment results

\begin{tabular}{l|cccccc} 
Test Area & Mean & Median & Std.Dev & NMAD & LE68 & LE95 \\
\hline Terrassa & $-0,04$ & $-0,26$ & 3,51 & 1,11 & 1,25 & 7,41 \\
Vacarisses & $-0,43$ & $-0,83$ & 4,74 & 2,05 & 2,35 & 10,23 \\
La Mola & $-0,87$ & $-1,12$ & 5,89 & 2,86 & 3,31 & 12,16
\end{tabular}

Table 2: Worldview-1 assessment results

- $\widetilde{\Delta Z}$ : median value of the height discrepancies $\Delta Z$

- Std.Dev: standard deviation of the height discrepancies $\Delta Z$ Std.Dev $=\sqrt{\frac{1}{N} \sum_{i=1}^{N}\left(\Delta Z_{i}-\overline{\Delta Z}\right)^{2}}$

- NMAD: Normalized Median Deviation $N M A D=1.4826 *$ median $(|\Delta Z-\widetilde{\Delta Z}|)$

- LE68: Linear error with $68 \%$ of probability

- LE95: Linear error with $95 \%$ of probability

\subsection{RESULTS}

The results of the statistical assessment in the different tiles, both for Cartosat- 1 and Worldview-1 stereo pair, are reported in Table 1 and Table 2.

As regards Cartosat- 1 imagery, the DSM accuracy in terms of NMAD ranges from 4.4 to $6.3 \mathrm{~m}$, whereas the standard deviation values are higher in all the areas, highlighting that there are some outliers in the error distributions. The mean and median values are consistent each other, but, a part from the Vacarisses tile, high biases are shown: in the La Mola area a negative bias of about 7 $\mathrm{m}$ is present, while the Terrassa tile shows a positive bias of about $3 \mathrm{~m}$. Probably these differences are due to the different prevalent land cover of the two tiles.

As far as concerns Worldview-1, the DSM accuracy in terms of NMAD ranges from 1.1 to $2.8 \mathrm{~m}$ (an enough similar values to LE68), whereas the standard deviation ranges from 3.5 to $5.9 \mathrm{~m}$ : this discrepancy between standard deviation and NMAD reports that the error distribution is not gaussian. The mean values are close to 0, a part from La Mola tile, that also for Worldview-1 produces higher results.

Making a comparison between the two sensors investigated, a general better accuracy of Worldview-1-derived DSMs with respect to Cartosat- 1 is shown. This evidence was to be expected due to the better resolution and orientation model of Worldview-1 imagery. In Figure 4 the error map of the three tiles for both sensors, are reported. Terrassa tile shows the most complete DSM (both for Cartosat-1 and for Worldview-1 imagery), whereas in La Mola area both DSMs are characterized by a higher percentage of unfilled pixels.

\section{CONCLUSIONS}

The developed strategy for DSM generation from satellite imagery, implemented in a Free and Open Source Software, and already tested both with optical and SAR satellite imagery, allows to achieve accurate results in an efficient way. The results obtained on the ISPRS data are encouraging, showing a general better accuracy for the Worldview-1 dataset. As a matter of fact, as regards the LE68, it ranges from $4.5 \mathrm{~m}$ to $6.3 \mathrm{~m}$ for Cartosat1 imagery and from $1.2 \mathrm{~m}$ to $3.3 \mathrm{~m}$ for Worldview-1. Also as regards the NMAD, another robust statistical parameter, the values range from $4.4 \mathrm{~m}$ to $6.0 \mathrm{~m}$ for Cartosat-1-derived DSMs, and from $1.1 \mathrm{~m}$ to $2.9 \mathrm{~m}$ for Wordlview-1.

Moreover, with this small dataset ( 3 tiles of $4 \times 4 \mathrm{~km}$ ), a negligible processing time has been witnessed, highlighting DATE efficiency.

As a future work, these results could be compared, in terms of statistical parameters, with those obtained through different strategies by other authors (d'Angelo et al. 2011), also with reference to different land covers.

\section{ACKNOWLEDGEMENTS}

The authors thanks the data providers for the provision of the stereo data sets, namely: Digital Globe for the Worldview-1 data, Euromap for the Cartosat-1 data, and ICC Catalunya for the reference data.

\section{REFERENCES}

d'Angelo, P., Reinartz, P., 2010. Semi-global matching results on the ISPRS stereo matching benchmark International Archives of the Photogrammetry, Remote Sensing and Spatial Information Sciences, Volume XXXVIII-4/W19, ISPRS Hannover 2011 Workshop.

Di Rita, M., Nascetti, A., Crespi, M., 2017. Open source tool for DSMs gen- eration from high resolution optical satellite imagery: development and testing of an OSSIM plug-in. International Journal of Remote Sensing, doi: 10.1080/01431161.2017.1288305.

Dröppelmann, S., Hueting, M., Latour, S., van der Veen, M., 2010. "Stereo Vision using the OpenCV library." https://tjpstereovision.googlecode.com/hg- history/551f9b6e2e9549337e7c26b4bac6a9a69a6c509c/doc/verslag.pdf.

Fratarcangeli, F., Murchio, G., Di Rita, M., Nascetti, A. and Capaldo, P., 2016. Digital surface models from ziyuan-3 triplet: performance evaluation and accuracy assessment. International Journal of Remote Sensing, 37(15), pp. 3505-3531.

Gesch, D. B., M. J. Oimoen, and G. A. Evans. 2014. Accuracy Assessment of the U.S. Geological Survey National Elevation Dataset, and Comparison with Other Large-Area Elevation Datasets - SRTM and ASTER. U.S. Geological Survey Open-File Report. 1008: 10.

Reinartz, P., dAngelo, P., Krauß, T., Poli, D., Jacobsen, K. and Buyuksalih, G., 2010. Benchmarking and quality analysis of 


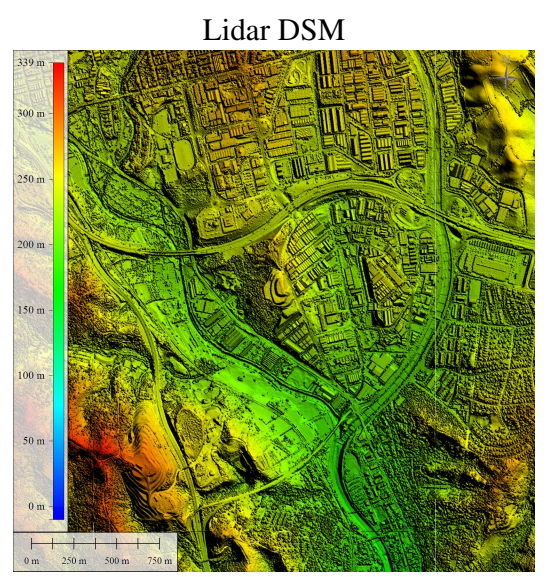

Lidar DSM

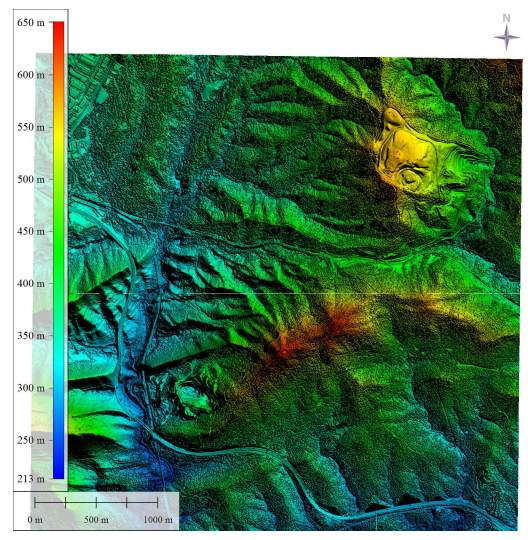

Lidar DSM

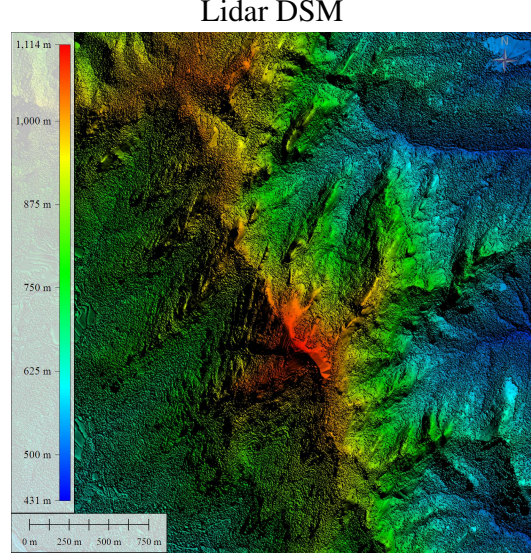

Terrassa area

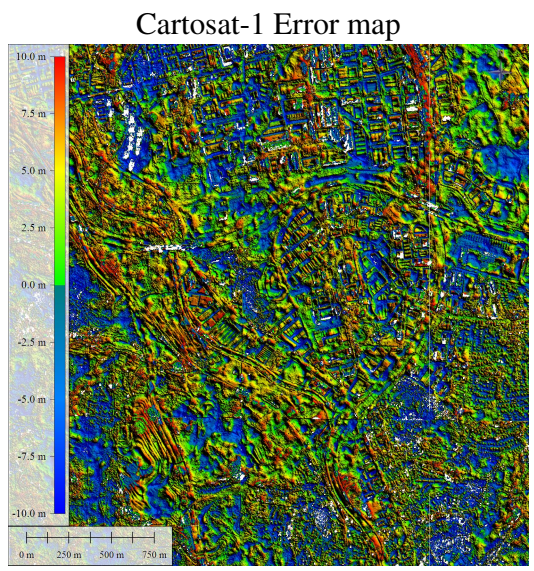

Vacarisses area

Cartosat-1 Error map

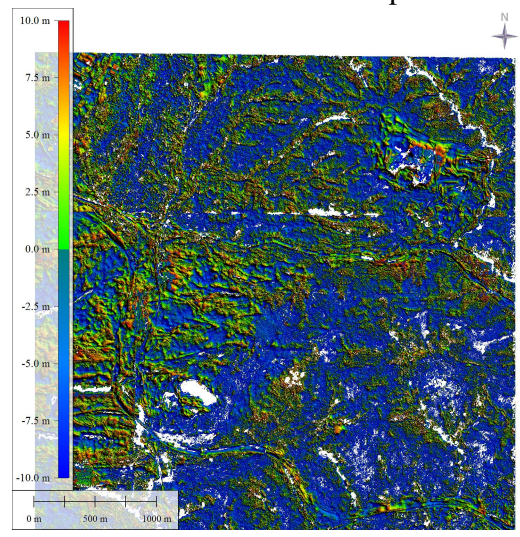

Lamola area

Cartosat-1 Error map

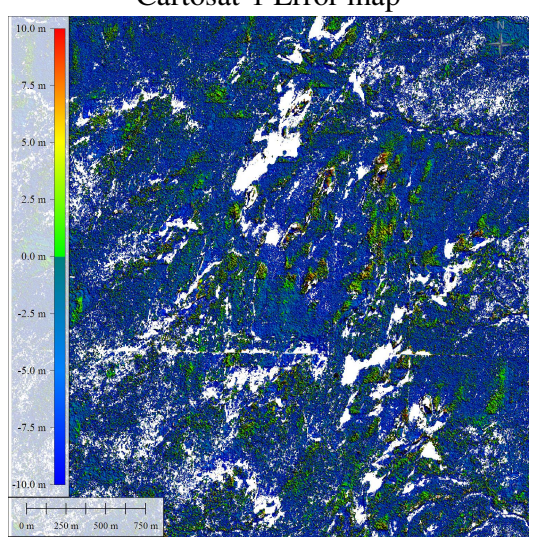

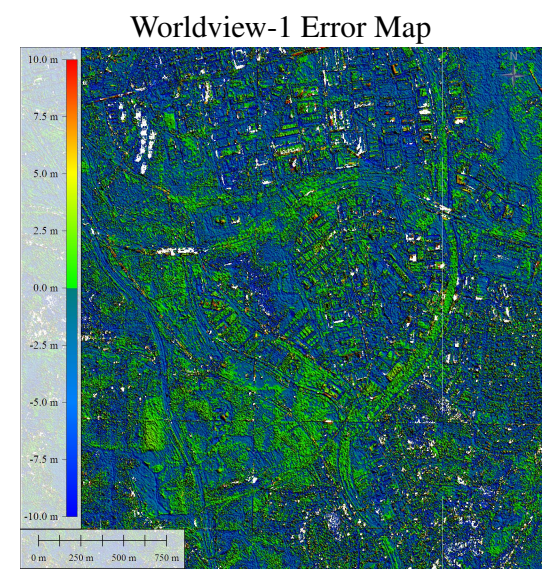

Worldview-1 Error Map

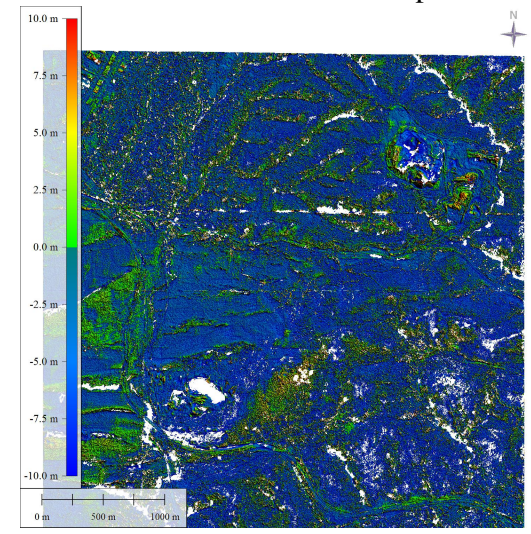

Worldview-1 Error Map

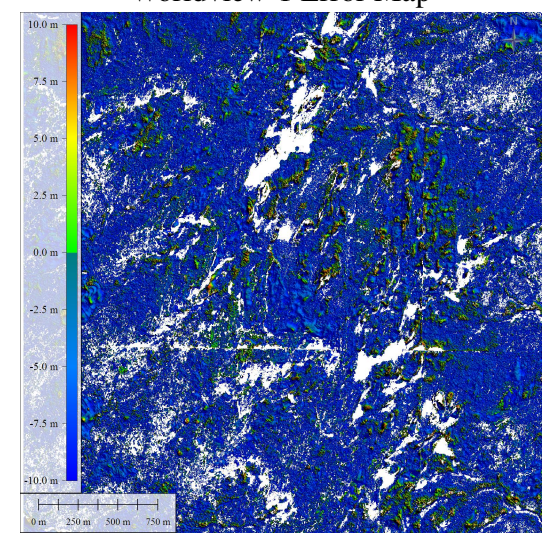

Figure 4: LiDAR reference DSMs and corresponding Error maps for the analysed tiles

dem generated from high and very high resolution optical stereo satellite data. ISPRS Symp. Commission I Int. Archives Photogrammetry Remote Sensing and Spatial Information Sciences, vol. XXXVIII.

Rodriguez, E., C. S. Morris, and E. Belz. 2006. A Global Assessment of the SRTM Performance. Photogrammetric Engineering and Remote Sensing, 72 (3): 249260. doi:10.14358/PERS.72.3.249. 